\title{
Urinary Exosome Based Androgen Receptor Variant 7 Detection in Metastatic Castration Resistant Prostate Cancer Patient
}

\section{Chao Wang}

Beijing pinggu hospital, Beijing friendship hospital, Capital medical university

\section{Xiang Liu}

Echo biotech Co. Ltd, Beijing

\section{Hongyan Li}

Echo biotech Co. Ltd, Beijing

Libo Zhao

Echo biotech Co. Ltd, Beijing

\section{Guanyi Kong}

Echo biotech Co., Ltd, Beijing

Jing Chen

Echo biotech Co., Ltd, Beijing

\section{Zhi Li}

Echo biotech Co., Ltd, Beijing

Jianfei Qi

University of Maryland, School of Medicine, Baltimore

\section{Ye Tian}

Beijing friendship hospital, Capital medical university

Fengbo Zhang ( $\sim$ 13701319061@130.com)

Beijing friendship hospital, Capital medical university

\section{Research article}

Keywords: androgen receptor, splicing variant, castration resistant prostate cancer, abiraterone, exosome

Posted Date: July 20th, 2020

DOl: https://doi.org/10.21203/rs.3.rs-41550/v1

License: (c) (i) This work is licensed under a Creative Commons Attribution 4.0 International License. Read Full License

Version of Record: A version of this preprint was published at Translational Andrology and Urology on January 1st, 2021. See the published version at https://doi.org/10.21037/tau-21-1136. 


\section{Abstract}

BACKGROUND Androgen receptor variant 7 (AR-V7) detection provides important reference to the clinical management of abiraterone in metastatic castration-resistant PCa (mCRPC). We performed a noninvasive urinederived exosomal AR-V7 analysis in MCRPC patients.

METHODS A total of $34 \mathrm{mCRPC}$ patients were categorized into 16 patients responded to abiraterone (ABI-Sta group) and 18 were resistant to abiraterone (ABI-Res group). First morning urine collected from patients as well as healthy control were used for analysis. Exosomal RNA was isolated for AR-V7 detection, and the correlation between AR-V7 expression and the therapeutic effect of abiraterone was analyzed.

RESULTS Urine is ideal biological sample for exosome separation and androgen receptor full-length (AR-FL) analysis. Positive urine-derived exosomal AR-V7 were detected in $32.4 \%$ ( 11 of 34 ) mCRPC urine samples. Positive AR-V7 is more common in ABI-Res patients ( $50.0 \%$ in ABI-Res group vs. $12.5 \%$ in ABI-Sta patients respectively, $p=0.009)$ and associate with higher PSA progression rate and poorer overall survival $(p=0.0031,0.0012)$.

CONCLUSIONS Urine-derived exosome is an alternative option for liquid biopsy-based AR-V7 detection in prostate cancer. Urinary exosomal AR-V7 is a dynamic marker that may predict the response to abiraterone in mCRPC patients.

\section{Background}

Prostate cancer ( $\mathrm{PCa}$ ) remains the second most common malignant among men worldwide, which accounts for more than 100 thousand death each year ${ }^{1,2}$. Although androgen deprivation therapy (ADT) have dramatically improved overall survival, almost all PCa patients will develop resistance to ADT, leading to a lethal state known as metastatic castration resistant $\mathrm{PCa}(\mathrm{mCRPC})$, which is incurable at present stage ${ }^{3}$.

In many cases, the androgen receptor (AR) axis remains active during the development of $\mathrm{MCRPC}$, indicating that the survival and growth of tumors still rely on the downstream signaling of AR pathway ${ }^{4}$. Abiraterone acetate blocks the intratumorally synthesis of androgens by selectively inhibiting the function of CYP171A, a key enzyme in androgen biosynthesis, thus is favored in the treatment of $\mathrm{MCRPC}$ patients ${ }^{5,6}$. Significant improve of median overall survival (OS) of patients treated with abiraterone has been demonstrated in several clinical trials (i.e. COU-AA-3017 , COU-AA$302^{8}$ and so on). However, the clinical outcome of abiraterone presents inter-individual variability. Different pathological and molecular biomarkers have been used for diagnosis, staging, and monitoring of MCRPC response to abiraterone. Among them, the expression of constitutively active ligand-independent AR variant, such as androgen receptor variant 7 (AR-V7), has been supposed to be most common mechanism of abiraterone resistance. AR-V7 is derived from the pre-mRNA of AR full length (AR-FL) through alternative splice ${ }^{9,10}$. The result of ligand binding domain (LBD) deletion is the constituting activating the transcription of AR downstream target genes and CRPC progression. Positive AR-V7 in PCa is strongly associated with the resistance to AR signaling inhibitors and poor clinical prognosis ${ }^{11}$. Therefore, detection of AR-V7 is valuable in prognosis evaluation and determining following therapeutic agents ${ }^{12}$.

Sensitive and specific bioanalytical method are indispensable to implement AR-V7 into clinical practice. AR and its splicing variants could be detected in both tumor tissue and body fluids. Tumor tissue can be acquired by biopsy, which is invasive, costly, most importantly, the re-biopsy samples are unavailable or unanalyzable in many cases ${ }^{13}$. Circulating tumor cell (CTCs) based AR-V7 quantification is the most used liquid biopsy strategy so far ${ }^{14,15}$. 
Nevertheless, sample processing for CTC is time sensitive and requires highly specialized equipment. Additionally, this method is only available for CTC positive patients, while a considerable number of patients are excluded due to low CTC burden or technical inaccessibility. Most importantly, both single-site biopsies and the specific antibody captured CTC do not fully considered the heterogeneity of prostate cancer ${ }^{16}$. As an alternative approach, AR-V7 RNA could also be extracted from whole blood for next bioanalysis, but this approach may be affected by the instability of free RNA in blood and the large amount of contaminating RNA 17,18 .

To date, exosome derived biomarkers have revolutionized the diagnosis of multiple pathologies including cancer ${ }^{19}$. Exosomes are small membranous vesicles arranged from $30-150 \mathrm{~nm}$, that are secreted into body fluid (including blood, cerebrospinal fluid, urine, milk) by various organ cells. Such extracellular vesicles (EVs) contain functional proteins and nucleic acids derived from the organs they originate from, then be uptake by target cells and readjust its physiological processes ${ }^{20}$. Showing the molecular signature of their histogenesis, exosomes represents one of the research hotspots in cancer diagnosis, medication guidance and treatment monitoring ${ }^{21,22}$. Specific to prostate cancer, a prospective study with limited sample indicated the plasma-derived exosomal RNA is a reliable source of $A R-V 7$ that can predict the resistance to hormonal therapy in prostate cancer ${ }^{23}$. Urine is the most ubiquitous body fluid that could regenerate rapidly. Furthermore, a direct physical connection between prostate and ureter was existed, indicating the possibility and reliability to detect AR-V7 in exosome derived from urine, which has been demonstrated by Cho with a small series of patients ${ }^{24}$.

In present study a small series of MCRPC patients treated with abiraterone acetate were enrolled and the expression of urine-derived exosomal AR-V7 was detected. The aim of present study is to determine the association between clinical outcomes and urine-derived exosomal AR-V7.

\section{Methods}

\section{Patients and healthy donor selection}

The study was officially approved by the ethics committee of Beijing friendship hospital. Written informed consents were received from all participants before urine collection and data analysis. The framework of this project was reported in the schema graph in Fig. 1. A total of 34 participants with metastatic CRPC treated with abiraterone acetate were enrolled in this cross-sectional program. All-inclusive patients were histologically confirmed prostate adenocarcinoma and castration levels of serum testosterone ( $\leq 20 \mathrm{ng} / \mathrm{dl}$ ) were maintained by ADT. Urine samples were collected for exosomal isolation after mCRPC was determined before next-line treatment strategy. All participants were categorized into abiraterone stable (ABI-Sta) group and abiraterone resistant (ABI-Res) group as per the following definition of abiraterone resistance. Prior docetaxel chemotherapy and radiotherapy were permitted. All patients were followed-up and clinical data, including PSA progression-free survival and overall survival was compared according to AR-V7 status.

Concerning disease progression including PSA and/or radiography progression according to PCWG criteria, in present study, patients with abiraterone resistance were enrolled as follows: (1) Ongoing abiraterone treatment present with at least three continuous rising serum PSA levels measured $\geq 1$ week apart and/or radiography progression confirmed by CT/MRI/bone scan consistent with the Prostate Cancer Working Group-2 (PCWG2) guidelines; (2) Ongoing abiraterone treatment more than 3 months and PSA flare should be excluded; (3) Ongoing androgen derivative treatment with $\mathrm{GnRH}$ analog or bilateral orchiectomy with serum testosterone $\leq 20 \mathrm{ng} / \mathrm{dl}$. Prior docetaxel-based chemotherapy was permitted. 
Data of baseline characters and pretreatment outcomes including age, Gleason score, prior treatment history, metastasis status, Eastern Cooperative Oncology Group (ECOG) performance status, serum PSA, alkaline phosphatase (ALP), lactate dehydrogenase ( $L D H)$, and hemoglobin were collected. Serum PSA was measured in all patients at least once per month during treatment, and imaging evaluation were performed every 3 months or at the physicians' discretion.

A total of 20 healthy controls, including 9 males and 11 females ranged from 40-70 years old, were recruited and confirmed with no evidence of prostatic malignancy. The analysis of AR-V7 in primary/metastasis tumors was not performed because this is not part of clinical practice.

\section{Urine collection and isolation of exosome}

For urine sample processing, $30 \mathrm{ml}$ morning urine were collected in RNAse-free urine cup and restore in room temperature less than 4 hours, then centrifuged at 3,000 $\mathrm{g}$ for $30 \mathrm{~min}$ and the supernatant was transferred to a new $50 \mathrm{ml}$ centrifuge tube and kept at $-80^{\circ} \mathrm{C}$.

Exosomes are isolated from urine sample following a modified protocol as reported ${ }^{19}$. Briefly, $30 \mathrm{ml}$ of fresh urine were centrifuged at $3,000 \mathrm{~g}$ for $30 \mathrm{~min}$ to eliminate cell debris and insoluble particle. $40 \%$ PEG-6000 was added into urine to a final concentration of $8 \%$, homogeneous mixing and to rest overnight. Then, the PEG-urine complex was centrifuged at $5,000 \mathrm{~g}$ for $20 \mathrm{~min}$, the exosomal pellets were re-suspended in $100 \mathrm{uL}$ of $1 \times \mathrm{PBS}$ and stored at $-80^{\circ} \mathrm{C}$ for further analysis.

\section{Nanoparticle tracking analysis (NTA) and transmission electron microscopy (TEM)}

Vesicle suspensions with concentrations between $1 \times 10^{7} / \mathrm{ml}$ and $1 \times 10^{9} / \mathrm{ml}$ were examined using the ZetaView PMX 110 (Particle Metrix, Meerbusch, Germany) equipped with a $405 \mathrm{~nm}$ laser to determine the size and quantity of particles isolated. A video of $60-\mathrm{sec}$ duration was taken with a frame rate of $30 \mathrm{frames} / \mathrm{sec}$, and particle movement was analyzed using NTA software (ZetaView 8.02.28).20 $\mu$ l exosomes solution was placed on a copper mesh and incubated at room temperature for $10 \mathrm{~min}$. After washing with sterile distilled water, the exosome was contrasted by uranyl-oxalate solution for $1 \mathrm{~min}$. The sample was then dried for 2 min under incandescent light. The copper mesh was observed and photographed under a transmission electron microscope (JEOL-JEM1400, Tokyo, Japan).

\section{Immunoblotting and exosome confirmation}

Exosomes were lysed by RIPA lysis buffer, 30 ug total protein were boiled in $2 \times$ SDS-PAGE loading buffer for 5 min and used for immunoblotting. Protein samples were separated by SDS-PAGE in 4-20\% and transferred to PVDF membranes by using tank method. The primary antibodies used in this experiment were listed as follows: anti-CD63 (Santa Cruz Biotechnology, sc-59284), anti-Alix (Abcam, ab117600), anti-TSG101 (Abcam, ab125011).

\section{Urine-derived exosomal RNA isolation and qRT-PCR}


The total RNA of exosome was extracted using QIAzol Lysis Reagent (QIAGEN, 79306) according to the manufacturer's protocol. All the total RNA was converted to cDNA using Superscript II reverse transcriptase (Invitrogen Life Technologies, 18064-014) following the manufacturer's instructions. The AR-FL was used as an internal control. The abundance of AR-FL and AR-V7 were detected by TaqMan real-time RT-PCR, the sequence of primers and probes were described as below: AR-FL, forward primer 5'- CATCAAGGAACTCGATCGT-3', the forward primer 5'- GAACTGATGCAGCTCTCTC - 3', probe 5'-ACATCCTGCTCAAGACGCTCCT-3'. AR-V7, forward primer 5'CTGTGCGCCAGCAGAAAT - 3', the forward primer 5'- TCAGGGTCTGGTCATTTTGA - 3', probe. 5'TGTCCATCTTGTCGTCTTCG-3' 23 .

\section{Standard curve preparation}

Briefly, the cDNA of AR-FL or AR-V7 was inserted into vector pBM-8A. The insert was cut from the plasmid and in vitro transcription was performed. After RNA was extracted using QIAzol Lysis Reagent and concentration quantification by nanodrop, the RNA was diluted in log increments from $1 \times 10^{6} \mathrm{copies} / \mathrm{uL}$ to $10 \mathrm{copies} / \mathrm{uL}$. The we carried out TaqMan fluorescent quantitative PCR in different RNA concentration and collected corresponding CT values.

\section{Statistical analysis}

All $P$ values were carried out using the SPSS software ((SPSS Inc., Chicago, II, USA)). All error bars represent mean \pm standard deviation (SD) derived from three independent experiments. Continuous data are presented as mean \pm standard deviation (SD) and independent samples $t$-test were conducted for comparison. Categorical data were presented as frequency and percentage and were evaluated using chi-square test. Time-to-event outcomes (PSA/radiographic progression-free survival [PFS] and overall survival [OS]) were analysed by the Kaplan-Meier method, and survival-time differences were compared by the log-rank test. In all cases, $P$ values of $<0.05$ were defined as statistically significant deference.

\section{Results}

\section{Urine-derived exosomal AR-FL and AR-V7 RNA expression in heathy volunteer}

To address whether urine could be used for exosome analysis, we first performed exosome isolation by PEG precipitation from urine (30 $\mathrm{ml}$ each test). Three evidence proved that urine was an ideal biological sample for exosome enrichment. First, western blotting analysis for the assessment of the common markers of exosome, indicating a significantly selective enrichment of exosome protein marker CD63 and TSG101 in urine derived exosome (Figure. 2a). Second, morphological analysis by transmission electron microscope (TEM) showed a classical double membrane structure in exosomes collected from urine, with an average diameter $88.9 \pm 9.81 \mathrm{~nm}$ (Figure. 2b). Third, nanoparticle tracking analysis (NTA) showed a concentration of exosome around $5 \times 10^{10}$ particles/30 ml urine, an acceptable abundance for further protein and RNA analysis (Figure. 2c,d).

The investigational part of this study was the assessment of AR-V7 in urine exosome from mCRPC patients. First, we conducted a standard curve reflected to the copy number of AR-V7 and AR-FL in each PCR tube and tested the detection limit in our system. According our standard curve, we could detect as low as 10 copies AR-FL and AR-V7 plasmid DNA in each reaction (Figure. 3a,b). Then, we enrolled 20 healthy controls. Total exosomal RNA was extracted for reverse transcription and further real-time quantitative PCR (RT-PCR). We could successfully detect AF- 
FL in all the entire 20 urine exosome samples from healthy volunteer (a mean CT value of 30.85). The Ct value less than 37, the cutoff value for AR-V7 were set as 36 to avoid false positive (Figure. 3c). These results were logically, as AR-FL was mainly expressed in hormone deprivation state, but not in normal physiology condition. These results proved urine-derived exosome enriched prostate-associated genetic material and might be more suitable for the ARV7 detection.

\section{Clinical characteristics and the expression of AR-V7 on urine- derived exosomal RNA}

Overall, all the 34 samples were positive for AR-FL, with an average Ct value 31.55 (74 copies/14 ml) and 11 samples showed the positive expression of AR-V7 with a Ct value arranged from 33.02-37.42 (2.5-45 copies/14 ml). Baseline characteristics for the participants are reported in Table 1. A total of 16 patients were categorized into abiraterone stable (ABI-Sta) group and 18 patients were into abiraterone resistant (ABI-Res) group respectively as per inclusion criteria. Median follow-up time was 15.7 months (range: 3.0-21.0 months). During follow-up, our data identified patients progressed with PSA progression, radiography progression and both are in 55.56\% (10/18), $22.22 \%(4 / 18)$ and $22.22 \%$ (4/18) of $18 \mathrm{ABI}$-Res patients versus in $25.0 \%(4 / 16), 12.5 \%(2 / 16)$ and $62.5 \%$ (10/16) of $16 \mathrm{ABI}-\mathrm{Sta}$ patients respectively. Comparing with ABI-Sta group, patients in ABI-Res group had more aggressive features such as more prior docetaxel-based chemotherapy(33.33\% vs. 6.25\%), higher median initial PSA level (241.24 ng/ml vs. $84.22 \mathrm{ng} / \mathrm{ml})$, serum ALP (205.51U/I vs. 420.71U/I) and LDH (125.62U/I vs. 143.31U/I) respectively. Positive AR-V7 was more common in ABI-Res patients (50.0\%) than that of ABI-Sta group (12.5\% in $\mathrm{ABI}$ Sta $V s 50.0 \%$ in ABI-Res, $P=0.009$ ) (Figure. $4 \mathrm{a}, \mathrm{b}$ ). In addition, the present of visceral metastasis is more common in ABI-Res group (7/18, 38.89\%), especially in ABI-Res AR-V7(+) subgroup (5/9, 55.56\%). 
Table 1. Characteristics of patients and androgen receptor splice variant 7 (AR-V7) status

\begin{tabular}{|c|c|c|c|c|c|c|}
\hline & \multicolumn{3}{|l|}{ ABI Stable } & \multicolumn{3}{|l|}{ ABI Resistant } \\
\hline & $\begin{array}{l}\text { ABI-Sta } \\
\text { SUM }\end{array}$ & AR-V7+ & AR-V7 - & $\begin{array}{l}\text { ABI-Res } \\
\text { SUM }\end{array}$ & AR-V7 + & AR-V7 - \\
\hline & $\mathrm{n}=16$ & $\mathrm{n}=2$ & $n=14$ & $n=18$ & $n=9$ & $n=9$ \\
\hline $\begin{array}{l}\text { Age, median } \\
\text { (range), y }\end{array}$ & 77.7 (59-85) & $77.5 \otimes 70-85 \rrbracket$ & $77.7 \otimes 59-80 \rrbracket$ & $72.9(61-83)$ & $72.6 \llbracket 61-83 \rrbracket$ & $73.2 \otimes 67-81 \rrbracket$ \\
\hline \multicolumn{7}{|l|}{$\begin{array}{l}\text { ECOG } \\
\text { performance } \\
\text { status, } n(\%)\end{array}$} \\
\hline 0 & 15 (93.75) & $2(100)$ & $13(92.86)$ & $14(77.78)$ & 7 (77.78) & 7 (77.78) \\
\hline 1 & $1(6.25)$ & $0(0)$ & $1(7.14)$ & $4(22.22)$ & $2(22.22)$ & $2(22.22)$ \\
\hline $\begin{array}{l}\text { Initial PSA at } \\
\text { diagnosis, } \\
\text { median } \\
\text { (range),ng/ml }\end{array}$ & $\begin{array}{l}84.22 \\
(10.12- \\
219.847)\end{array}$ & $\begin{array}{l}24.93 \otimes 10.12- \\
39.73 \rrbracket\end{array}$ & $\begin{array}{l}92.69 \otimes 12.0- \\
219.847 \rrbracket\end{array}$ & $\begin{array}{l}241.24 \\
(24.76- \\
536.0)\end{array}$ & $\begin{array}{l}275.42 \\
\varangle 42.0-536.0 \rrbracket\end{array}$ & $\begin{array}{l}207.05 \\
(24.76- \\
501.0)\end{array}$ \\
\hline $\begin{array}{l}\text { Baseline ALP, } \\
\text { median } \\
\text { (range),U/I }\end{array}$ & $\begin{array}{l}205.51(58- \\
352)\end{array}$ & $\begin{array}{l}145.0(65- \\
225)\end{array}$ & $\begin{array}{l}214.15(58- \\
352)\end{array}$ & $\begin{array}{l}420.71(77- \\
2210)\end{array}$ & $\begin{array}{l}447.25(134- \\
2210)\end{array}$ & $\begin{array}{l}394.20(77- \\
1401)\end{array}$ \\
\hline $\begin{array}{l}\text { Baseline LDH, } \\
\text { median } \\
\text { (range),U/I }\end{array}$ & $\begin{array}{l}125.61(76- \\
200)\end{array}$ & $\begin{array}{l}155.0(110- \\
200)\end{array}$ & $\begin{array}{l}132.50(76- \\
177)\end{array}$ & $\begin{array}{l}143.31(82- \\
250)\end{array}$ & $\begin{array}{l}152.18(101- \\
250)\end{array}$ & $\begin{array}{l}134.44(82- \\
245)\end{array}$ \\
\hline $\begin{array}{l}\text { Baseline Hgb, } \\
\text { median } \\
\text { (range),g/dl }\end{array}$ & $\begin{array}{l}108.90(93.0- \\
134.0)\end{array}$ & $\begin{array}{l}\text { 118.32(110.1- } \\
126.5)\end{array}$ & $\begin{array}{l}107.56(93.0- \\
134.0)\end{array}$ & $\begin{array}{l}106.63(88.5- \\
133.0)\end{array}$ & $\begin{array}{l}104.50(88.5- \\
129.0)\end{array}$ & $\begin{array}{l}108.76(93.0- \\
133.0)\end{array}$ \\
\hline \multicolumn{7}{|l|}{$\begin{array}{l}\text { Type of local } \\
\text { therapy, } n(\%)\end{array}$} \\
\hline Surgery & $2(12.5)$ & $1 \otimes 50 \rrbracket$ & $1 \otimes 7.14 \rrbracket$ & $3(16.67)$ & $1 \otimes 11.11 \rrbracket$ & $2 \rrbracket 22.22 \rrbracket$ \\
\hline Branchytherapy & $2(12.5)$ & 0 & $2(14.29)$ & 0 & 0 & 0 \\
\hline None & $12(75.0)$ & $1 \rrbracket 50 \rrbracket$ & $11 \otimes 78.57 \rrbracket$ & 15 (83.33) & $8 \bowtie 88.9 \rrbracket$ & $7 \rrbracket 77.78) \rrbracket$ \\
\hline \multicolumn{7}{|l|}{$\begin{array}{l}\text { Prior use of } \\
\text { docetaxel, } n(\%)\end{array}$} \\
\hline Yes & $1(6.25)$ & $0 \triangle 0 \bigotimes$ & $187.14 \rrbracket$ & $6(33.33)$ & $3 \rrbracket 33.3 \rrbracket$ & $3 \rrbracket 33.3 \rrbracket$ \\
\hline No & 15 ( 93.75) & $2 \otimes 100 \rrbracket$ & $13 \varangle 92.86 \rrbracket$ & $12(66.67)$ & $6 \rrbracket 66.7 \rrbracket$ & $6 \rrbracket 66.7 \rrbracket$ \\
\hline \multicolumn{7}{|l|}{$\begin{array}{l}\text { Gleason score } \\
\text { at diagnosis }\end{array}$} \\
\hline 7 & $11(68.75)$ & $1(50.0)$ & $10(71.43)$ & $4(22.22)$ & $1(11.11)$ & $3(33.33)$ \\
\hline$\geq 8$ & $5(31.25)$ & $1(50.0)$ & $4(28.57)$ & $14(77.78)$ & $8(88.89)$ & $6(66.67)$ \\
\hline \multicolumn{7}{|l|}{$\begin{array}{l}\text { Prior use of } \\
\text { radiotherapy, } \\
n(\%)\end{array}$} \\
\hline Yes & $3(18.75)$ & $1 \otimes 50 \rrbracket$ & $2 \otimes 14.29 \rrbracket$ & $6(33.33)$ & 3 (33.3) & $3(33.3)$ \\
\hline
\end{tabular}




\begin{tabular}{|c|c|c|c|c|c|c|}
\hline No & $13(81.25)$ & $1 \otimes 50 \rrbracket$ & $12(85.71)$ & $12(66.67)$ & $6(66.67)$ & $6(66.67)$ \\
\hline \multicolumn{7}{|l|}{$\begin{array}{l}\text { Presence of } \\
\text { bone } \\
\text { metastases, } \\
\text { n(\%) }\end{array}$} \\
\hline Yes & $16(100)$ & $2 ه 100 \rrbracket$ & 12ه100》 & 18 (100) & $8 \otimes 100 \rrbracket$ & 10ه100》 \\
\hline \multicolumn{7}{|l|}{$\begin{array}{l}\text { Presence of } \\
\text { visceral } \\
\text { metastases, } \\
\text { n(\%) }\end{array}$} \\
\hline Yes & $1(6.25)$ & $0(0)$ & $1(7.14)$ & 7 (38.89) & $5(55.56)$ & $2(22.22)$ \\
\hline No & 15 (93.75) & $2(100)$ & $13(92.86)$ & $11(61.11)$ & $4(44.44)$ & 7 (77.78) \\
\hline $\begin{array}{l}\text { Time to CRPC, } \\
\text { median (range), } \\
\text { month }\end{array}$ & $43.1(9-117)$ & $42.5(30-55)$ & $\begin{array}{l}41.17(9- \\
117)\end{array}$ & $31.7(9-110)$ & $27.8(9-110)$ & $35.7(9-106)$ \\
\hline
\end{tabular}

To further analysis, both groups were categorized into AR-V7(+) and AR-V7(-) subgroups respectively according to ARV7 analysis. Subgroup data shows that patients in ABI-Res AR-V7(+) subgroup have more invasive disease with higher initial PSA level at diagnosis $(275.42 \mathrm{ng} / \mathrm{dl}$ vs. $207.05 \mathrm{ng} / \mathrm{dl})$ and shorter ADT time to CRPC ( $27.75 \mathrm{mo}$ vs $35.67 \mathrm{mo}$ ) comparing with that of ABI-Res AR-V7(-) subgroup.

Kaplan-Meier analysis did not confirmed the role of AR-V7 as an independent prognostic factor as for overall survival (hazard ratio: $1.645,95 \% \mathrm{Cl}, 0.611-4.428, P=0.3089$ )and PSA progression-free survival (hazard ratio: $1.821,95 \%$ $\mathrm{Cl}, 0.797-4.162, P=0.1133$ ) probably due to the small series patients enrolled (Fig. 5). However, analyzing at the 20th months timepoint displayed there are 10/11 (90.91\%) positive AR-V7 patients experienced an increase of $\geq 50 \%$ in PSA response and/or radiography progression versus $14 / 23(60.87 \%)$ in negative AR-V7 patients $(p=0.0031$, 0.0012).

\section{Discussion}

The alternative splicing AR-V7 was reported to couple with AR gene transcription and facilitated by AR gene amplification/rearrangement. The positive expression of AR-V7 associates with the resistance to therapeutic target to AR pathway (abiraterone and enzalutamide) in CRPC patients. Detecting of the expression of AR-V7 is vital to decide the following treatment strategy of CRPC patients. Unfortunately, re-biopsy to obtain prostate cancer tissue is often unacceptable in clinical practice. More clinicians refer to liquid biopsy to maintain the detection, which acquired multiple advantages of noninvasive, biomaterial availability, and could represent tumor heterogeneity. Blood sample derived RNA, both from CTC and exosome, have been used to interrogate tumor biology. Nevertheless, the clinical value of AR-V7 detection in urine remains deeply uncertain, which was tested in an individual study with small number of samples ${ }^{24}$. The feasibility of EVs isolation from urine was confirmed in present study. Here, we describe our workflow of urine exosome extraction and exosomal AR-V7/AR-FL detection using real-time quantitative PCR instead of ddPCR. Healthy controls were enrolled to definite the cut-off values of AR-V7/AR-FL. Next, we enrolled mCRPC patients treated with abiraterone to explore the correlation between the urine exosomal-derived AR-V7 and 
therapeutic effect of abiraterone in MCRPC. Our data displayed AR-V7 status in urine-derived exosome is highly associate to $\mathrm{mCRPC}$ clinical prognosis. Positive AR-V7 was more common in patients of abiraterone resistant group than that of abiraterone stable group. Moreover, positive AR-V7 seems to relate to higher initial PSA level at diagnosis, Gleason scores, shorter ADT time to CRPC and survival.

Marzia et al investigated the expression of AR-V7 on plasma-derived exosome previously. In that small CRPC series (36 patients) before they began second-line hormonal treatment, positive AR-V7 was detected in 39\% patients and associate with poor clinical prognosis ${ }^{23}$. It demonstrates the priorities of RNA recovered from plasma-derived exosomes could benefit the CRPC diagnostic workflow and minimize the negative effect of tumor heterogeneity. Given that efficient isolation of tumor-specific materials from whole blood remains a major challenge due to the interruption of abundance contaminants, such as cells, vesicles, and proteins from healthy tissues, more sensitive and reliable liquid biopsy for AR-V7 detection is highly demanded. Woo HK et al. demonstrated their workflow of ARV7 mRNA expression in urinary EVs in prostate cancer patients using ddPCR assay. Although the clinic follow-up data was absent, it was observed the tendency for higher AR-V7 expression in urine-derived EVs from patients with CRPC than in those with hormone sensitive PCa ${ }^{24}$. Here, we present our work that AR-V7 RNA detection with isolated urine-derived exosomes using qRT-PCR assay. The $50 \%$ positive rate of AR-V7 on urine-derived exosomes in abiraterone resistant patients demonstrates a more sensitive detection technology for AR-V7. In theory, plasma samples contained large amount non-cancer related exosomes in plasma that secreted by leucocytes, platelet, red blood cell, which might lead to high frequency false positives detection of AR-V7. Nevertheless, most the biological macromolecule from blood could be excluded by the renal corpuscle, leaving the urine containing more biomolecules from prostate, as well as bladder. In this respect, urine derived exosomes showed certain advantages over blood derived exosomes in monitoring of urinary tract related cancers such as prostate cancer. Initially, we supposed most EVs secreted from shedding cells of urethra epithelium which may contains more EVs of prostate cancer cells until the two positive AR-V7 patients were figured out with prior radical proctectomy. It confirms the reasonability that exosomes may be secreted by renal corpuscle or urothelial cells.

Moreover, we should acknowledge that it is not a prospective designed randomized research and only a small series of patient were enrolled. Our Kaplan-Meier analysis did not reach significant difference according to OS and PFS probably due to the limited patients enrolled. However, analysis of PSA progression and/or radiography progression rates at the 20th months timepoint showed positive AR-V7 seems to associate with higher PSA progression and/or radiography progression rates (90.91\% vs. 60.87\%). A large-scale prospective trail is now required to evaluate this pilot study.

Despite the elevated AR-V7 positive proportion in this series, still 50.0\% abiraterone resistant CRPC patients were absent of AR-V7 expression. These results remind the diversity of the molecular mechanism under abiraterone tolerance. Other alternatively spliced transcripts of androgen receptor, such as ARV3, ARV9 could also be involved in the development of abiraterone resistance. According to our results, urine-derived exosome contained abundant molecular information that relevant to prostatic cancer tissues, which could be further used for biomarker screening related to abiraterone resistance. Last, the present study mainly enrolled CRPC patients treated with abiraterone, due to the unreachable of enzalutamide and a similar conclusion cannot be applied in patients treated with enzalutamide.

\section{Conclusions}


The present study demonstrates the detection of urine-derived exosomal AR-V7 is a more sensitive and feasible clinical workflow without any invasiveness. The role of urine-derived exosomal AR-V7 in mCRPC patients should be further verified by more sample size research. AR-FL: Androgen receptor full-length; LBD: Ligand binding domain;

\section{Abbreviations}

Pca: Prostate cancer; CRPC: Castration resistant prostate cancer; AR: Androgen receptor; AR-V7: Androgen receptor variant 7; ADT: Androgen deprivation therapy; CDC: Circulating tumor cell; EV: Extracellular vesicle; ECOG: Eastern Cooperative Oncology Group; ALP: Alkaline phosphatase; LDH: Lactate dehydrogenase; NTA: Nanoparticle tracking analysis; TEM: Transmission electron microscopy; PFS Progression-free survival; OS: Overall survival.

\section{Declarations}

\section{Ethics approval and consent to participate}

The study was officially approved by the ethics committee of Beijing friendship hospital. Written informed consents were received from all participants before urine collection and data analysis.

\section{Consent for publication}

Not applicable.

\section{Funding}

Dr. Fengbo Zhang received financial support from Beijing natural science foundation $₫ 7192044 \rrbracket$ which was used to maintain the exosome isolation, AR-V7 analysis and manuscript review. Dr. Chao Wang is funded by Beijing pinggu hospital research foundation (pgyyqn2019-03) which used to supported his role in study data analysis and patient management.

\section{Availability of data and material}

The datasets used and/or analyzed during the current study are available from the corresponding author on reasonable request.

\section{Authors' contributions}

FBZ and GYK study conception and design. CW, XL and JC study coordinator, exercise trainer, outcome assessor, data manager, data analysis. CW and HYL study management. FBZ and CW study physicians and medical advice. LBZ, GYK and ZL statistical analyses. FBZ, YT and JFQ drafted and finalized the manuscript. All authors have read and approved the final manuscript.

\section{Competing interests}

All authors declare no potential competing interest. 


\section{Acknowledgements}

The authors wish to thank Prof. Bangwei Cao for providing his constructive advice during protocol design, Dr. Min Cong for recruitment assistant, and all the study participants for their involvement.

\section{References}

1. Siegel RL, Miller KD, Jemal A.Cancer statistics, 2018. CA: a cancer journal for clinicians. 2018;68(1): 7-30. doi:10.3322/caac. 21442

2. Skolarus, TA, Wolf AM, Erb NL, Brooks DD, Rivers BM, Underwood W 3rd, et al. American Cancer Society prostate cancer survivorship care guidelines. CA: a cancer journal for clinicians. 2014; 64(4): 225-49. doi:10.3322/caac.21234.

3. Grasso, CS. Robinson DR, Cao X, Dhanasekaran SM, Khan AP. et al. The mutational landscape of lethal castration-resistant prostate cancer. Nature. 2012;487(7406), 239-43. doi:10.1038/nature11125.

4. Zadra, G, Ribeiro CF, Chetta P, Ho Y, Cacciatore S, Gao X, et al. Inhibition of de novo lipogenesis targets androgen receptor signaling in castration-resistant prostate cancer. Proceedings of the National Academy of Sciences of the United States of America. 2019; 116(2): 631-40. doi:10.1073/pnas.1808834116.

5. Li Z, Bishop AC, Alyamani M, Garcia JA, Dreicer R, Bunch D, et al. Conversion of abiraterone to D4A drives antitumour activity in prostate cancer. Nature. 2015; 523(7560): 347-51. doi:10.1038/nature14406.

6. Li Z, Alyamani M, Li J, Rogacki K, Abazeed M, Upadhyay SK, et al. Redirecting abiraterone metabolism to finetune prostate cancer anti-androgen therapy. Nature. 2016; 533(7640), 547-51. doi:10.1038/nature17954.

7. S. de Bono, C.J. Logothetis, A. Molina, et al. Abiraterone and increased survival in metastatic prostate cancer. $\mathrm{N}$ Engl J Med, 2011; 364 (21): 1995-2005. doi: 10.1056/NEJMoa1014618.

8. J. Ryan, M.R. Smith, J.S. de Bono, et al. Abiraterone in metastatic prostate cancer without previous chemotherapy.N Engl J Med, 2013; 368 (2): 138-148. doi: 10.1056/NEJMoa1209096.

9. Tsaur, I., Becker, C., Thelen, P. \& Roos, F. C. [Splice variant AR-V7 : Is it time for its routine use as a predictive marker in prostate cancer?]. Der Urologe. Ausg. A. 2017;56(9), 1164-7. doi:10.1007/s00120-017-0461-x.

10. Sharp A, Coleman I, Yuan W, Sprenger C, Dolling D, Rodrigues DN, et al. Androgen receptor splice variant-7 expression emerges with castration resistance in prostate cancer. The Journal of clinical investigation. 2018;129(1):192-208. doi:10.1172/JCl122819.

11. Navarro HI, Goldstein AS. HoxB13 mediates AR-V7 activity in prostate cancer. Proceedings of the National Academy of Sciences of the United States of America. 2018;115(26): 6528-9. doi:10.1073/pnas.1808196115.

12. Markowski MC, Silberstein JL, Eshleman JR, Eisenberger MA, Luo J, Antonarakis ES. Clinical Utility of CLIAGrade AR-V7 Testing in Patients With Metastatic Castration-Resistant Prostate Cancer. JCO precision oncology. 2017, Epub 2017 Oct 30 doi:10.1200/P0.17.00127 (2017).

13. Scher HI, Graf RP, Schreiber NA, McLaughlin B, Lu D, Louw J, et al. Nuclear-specific AR-V7 Protein Localization is Necessary to Guide Treatment Selection in Metastatic Castration-resistant Prostate Cancer. European urology. 2017;71(6):874-82. doi:10.1016/j.eururo.2016.11.024.

14. Tommasi S, Pilato B, Carella C, Lasorella A, Danza K, Vallini I, et al. Standardization of CTC AR-V7 PCR assay and evaluation of its role in castration resistant prostate cancer progression. The Prostate. 2019;79(1): 54-61. doi:10.1002/pros.23710. 
15. Scher HI, Lu D, Schreiber NA, Louw J, Graf RP, Vargas HA, et al. Association of AR-V7 on Circulating Tumor Cells as a Treatment-Specific Biomarker With Outcomes and Survival in Castration-Resistant Prostate Cancer. JAMA oncology. 2016,2(11): 1441-9, doi:10.1001/jamaoncol.2016.1828.

16. Ignatiadis M, Lee M, Jeffrey SS. Circulating Tumor Cells and Circulating Tumor DNA: Challenges and Opportunities on the Path to Clinical Utility. Clinical cancer research. 2015.21(21): 4786-800. doi:10.1158/10780432.CCR-14-1190.

17. Seitz AK, Thoene S, Bietenbeck A, Nawroth R, Tauber R, Thalgott M, et al. AR-V7 in Peripheral Whole Blood of Patients with Castration-resistant Prostate Cancer: Association with Treatment-specific Outcome Under Abiraterone and Enzalutamide. European urology. 2017; 72(5), 828-34. doi:10.1016/j.eururo.2017.07.024.

18. To SQ, Kwan EM, Fettke HC, Mant A, Docanto MM, Martelotto L, et al. Expression of Androgen Receptor Splice Variant 7 or 9 in Whole Blood Does Not Predict Response to Androgen-Axis-targeting Agents in Metastatic Castration-resistant Prostate Cancer. European urology. 2018; 73(6): 818-21. doi:10.1016/j.eururo.2018.01.007.

19. Tkach M, Thery C. Communication by Extracellular Vesicles: Where We Are and Where We Need to Go. Cell. 2016;164(6): 1226-32. doi:10.1016/j.cell.2016.01.043.

20. Houali K, Wang X, Shimizu Y, Djennaoui D, Nicholls J, Fiorini S, et al. A new diagnostic marker for secreted Epstein-Barr virus encoded LMP1 and BARF1 oncoproteins in the serum and saliva of patients with nasopharyngeal carcinoma. Clinical cancer research. 2007;13(17): 4993-5000. doi:10.1158/1078-0432.CCR-062945.

21. Castellanos-Rizaldos E, Grimm DG, Tadigotla V, Hurley J, Healy J, Neal PL, et al. Exosome-Based Detection of EGFR T790M in Plasma from Non-Small Cell Lung Cancer Patients. Clinical cancer research. 2018;24(12): 294450. doi:10.1158/1078-0432.CCR-17-3369.

22. Kannan A, Wells RB, Sivakumar S, Komatsu S, Singh KP, Samten B, et al. Mitochondrial Reprogramming Regulates Breast Cancer Progression. Clinical cancer research. 2016;22(13): 3348-60. doi:10.1158/10780432.CCR-15-2456.

23. Del Re M, Biasco E, Crucitta S, Derosa L, Rofi E, Orlandini C, et al. The Detection of Androgen Receptor Splice Variant 7 in Plasma-derived Exosomal RNA Strongly Predicts Resistance to Hormonal Therapy in Metastatic Prostate Cancer Patients. European urology. 2017; 71(4): 680-7. doi:10.1016/j.eururo.2016.08.012.

24. Woo HK, Park J, Ku JY, Lee CH, Sunkara V, Ha HK, et al. Urine-based liquid biopsy: non-invasive and sensitive ARV7 detection in urinary EVs from patients with prostate cancer. Lab Chip.2018;19(1):87-97. doi:

10.1039/c8lc01185k.

\section{Figures}




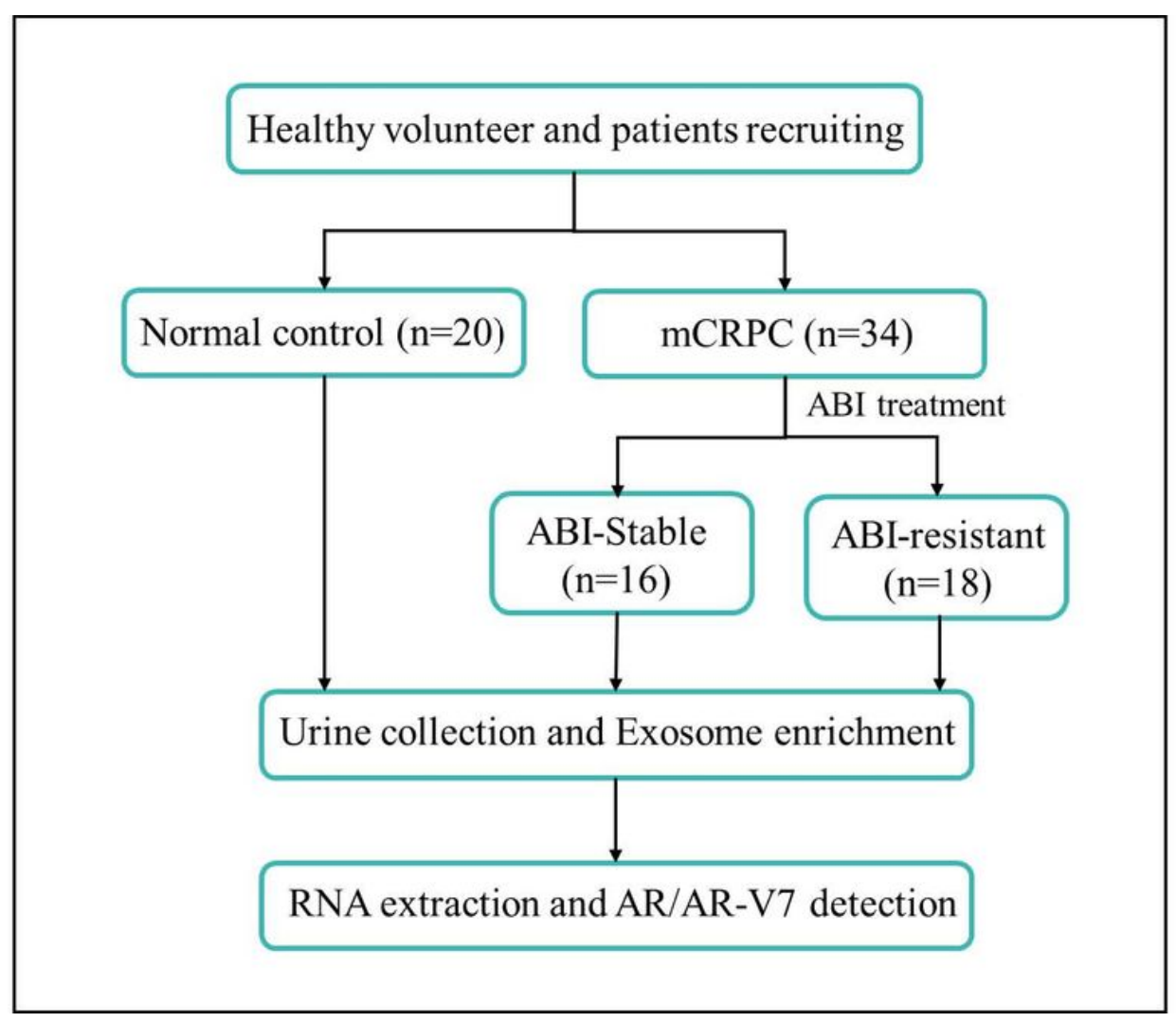

Figure 1

Framework of this project 
a
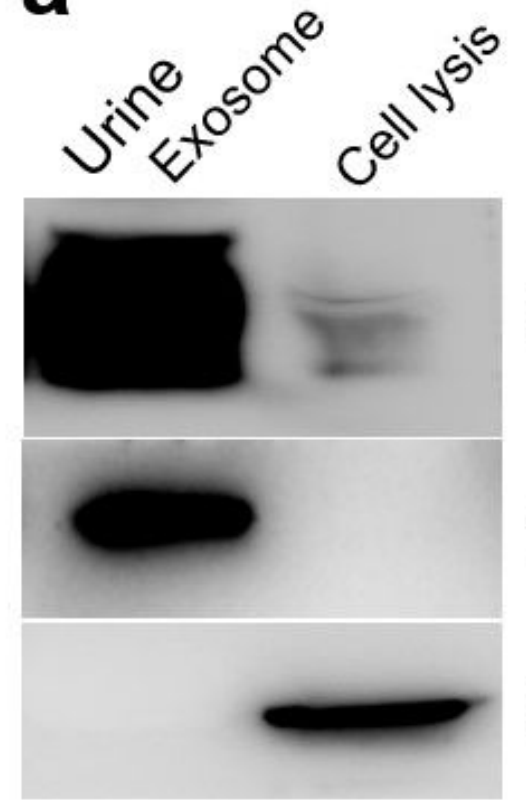

CD63

Tsg101

Calnexin

\section{C}

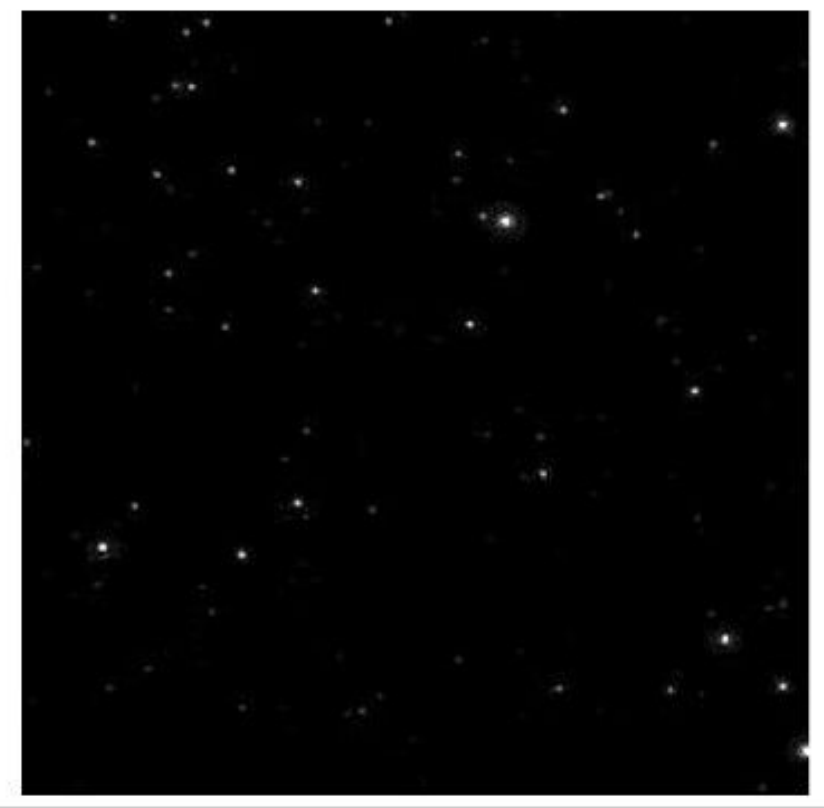

\section{Fig.2 \\ b}

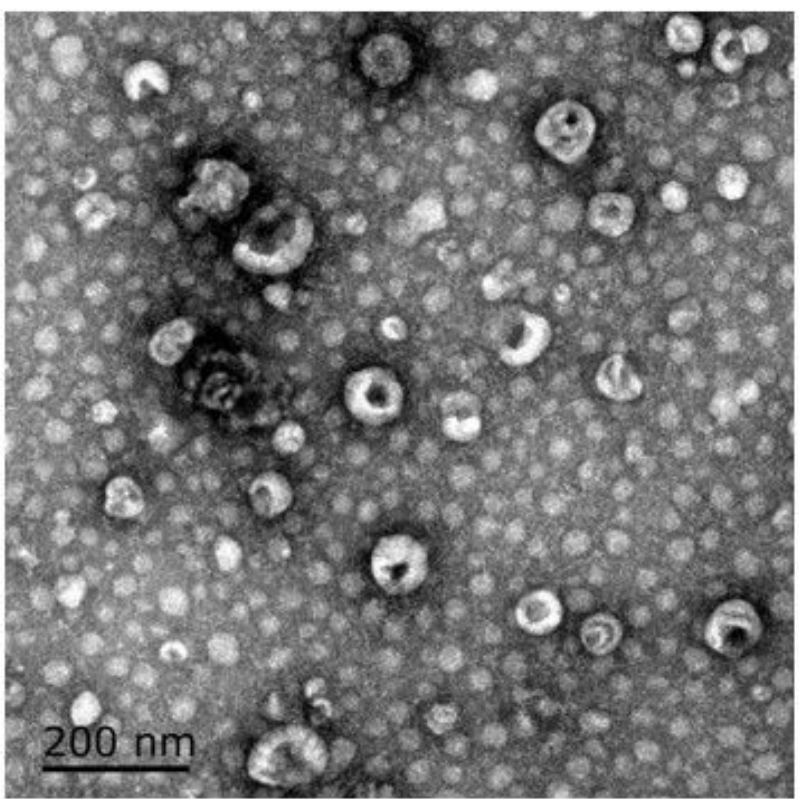

d

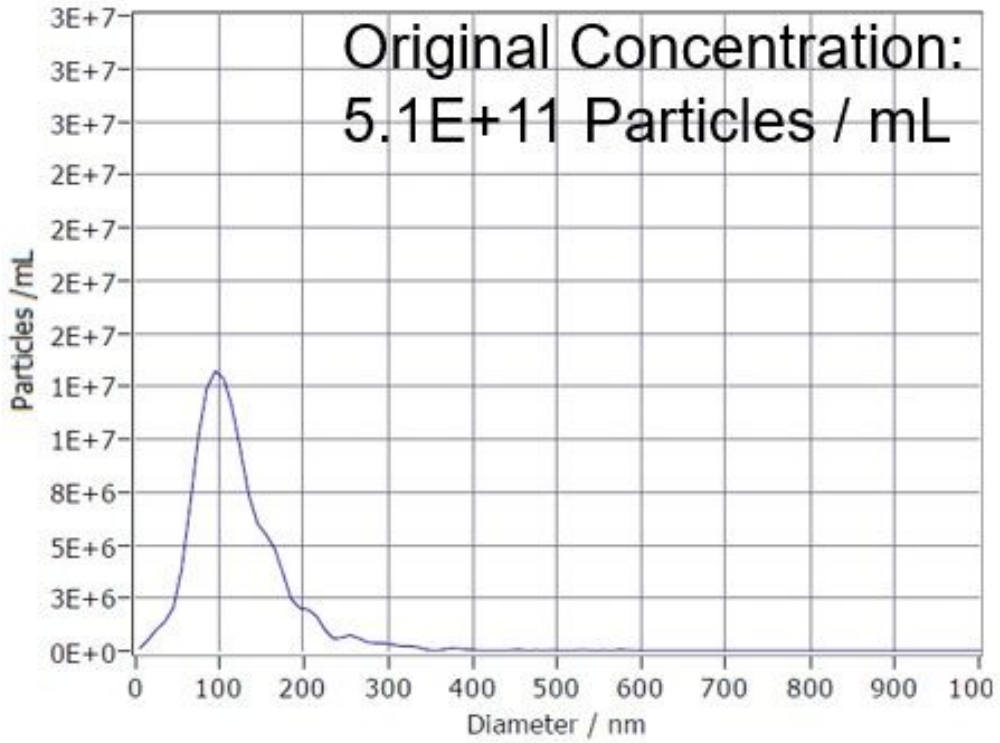

Figure 2

Exosome extraction from urine a. Immunoblotting of exosome marker CD63 and Tsg101 in exosomes derived from urine. Calnexin was a protein located cytoplasm but not in exosome was used as a negative control. b.

Representative TEM image of exosomes derived from urine. c,d. Representative NTA image of exosomes derived from urine (c), the size distribution and particle concentration was showed in $\mathrm{d}$. 


\section{Fig.3}

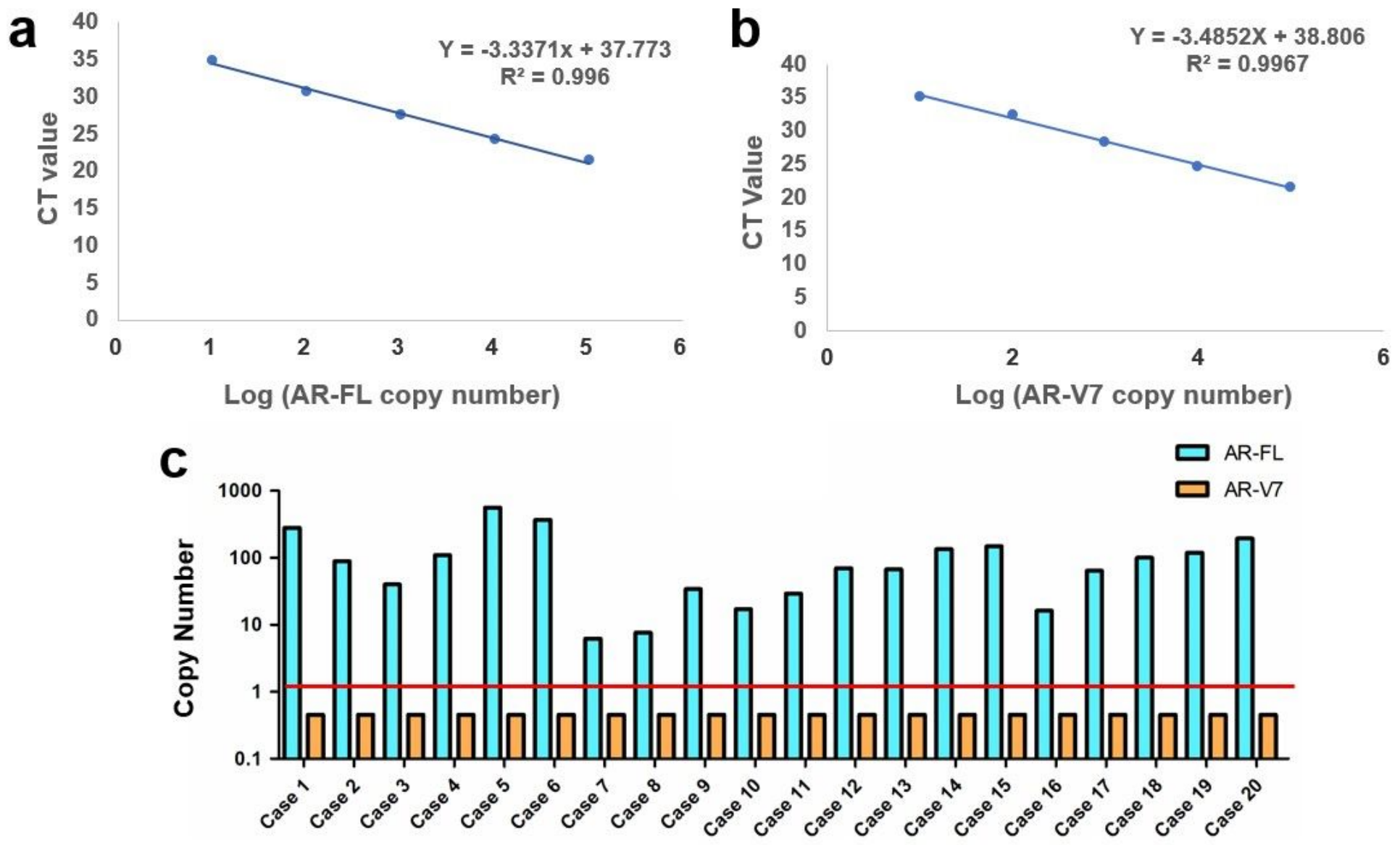

Figure 3

AR-FL/AR-V7 detection in urine of healthy volunteers a,b. Standard curve of AR-FL (a), AR-V7 (b) copy number and corresponding CT value in the real time-PCR quantification experiment. c. Quantification of the expression level of $\mathrm{AR}-\mathrm{FL}$ and $\mathrm{AR}-\mathrm{V} 7$ in exosomes derived from urine of healthy volunteers, the data was displayed as copy numbers/14 $\mathrm{ml}$ urine. Negative samples were assigned a Ct value of 40 , the cutoff line of AR-V7 positive was showed by red line. 
a

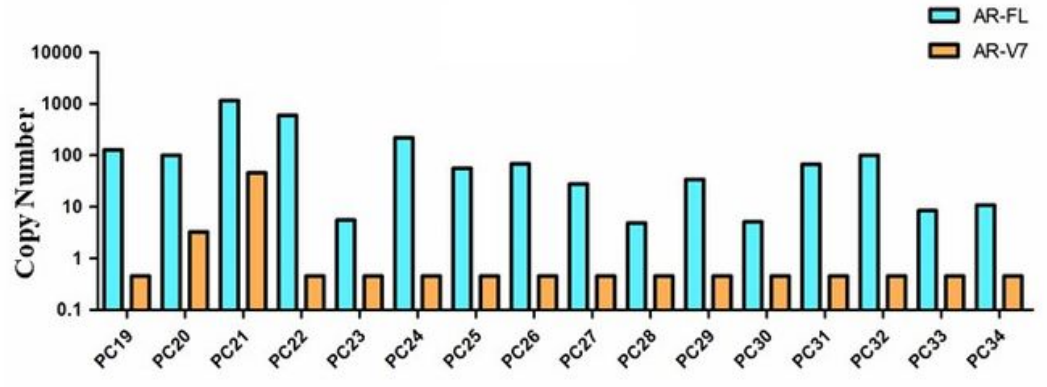

b

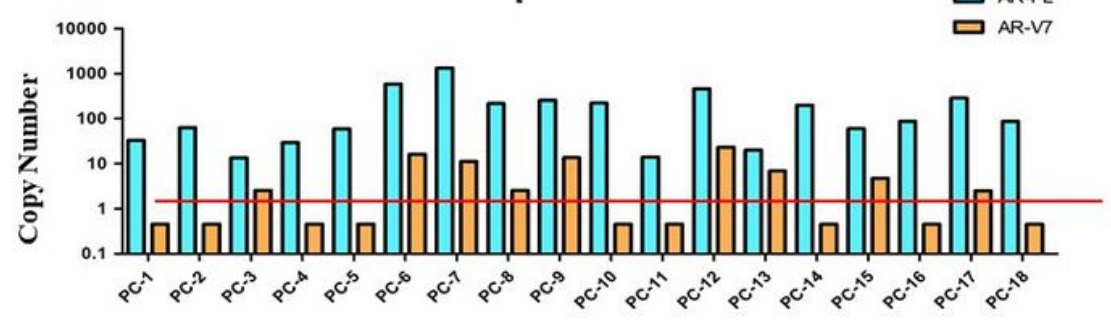

\section{Figure 4}

AR-FL/AR-V7 detection in urine-derived exosomes of prostate cancer a,b. Quantification of the expression level of AR$F L$ and $A R-V 7$ in exosomes derived from urine of $A B I$ stable group and $A B I$ Resistance group, the data was displayed as copy numbers $/ 14 \mathrm{ml}$ urine. Negative samples were assigned a Ct value of 40 , the cutoff line of AR-V7 positive was showed by red line.
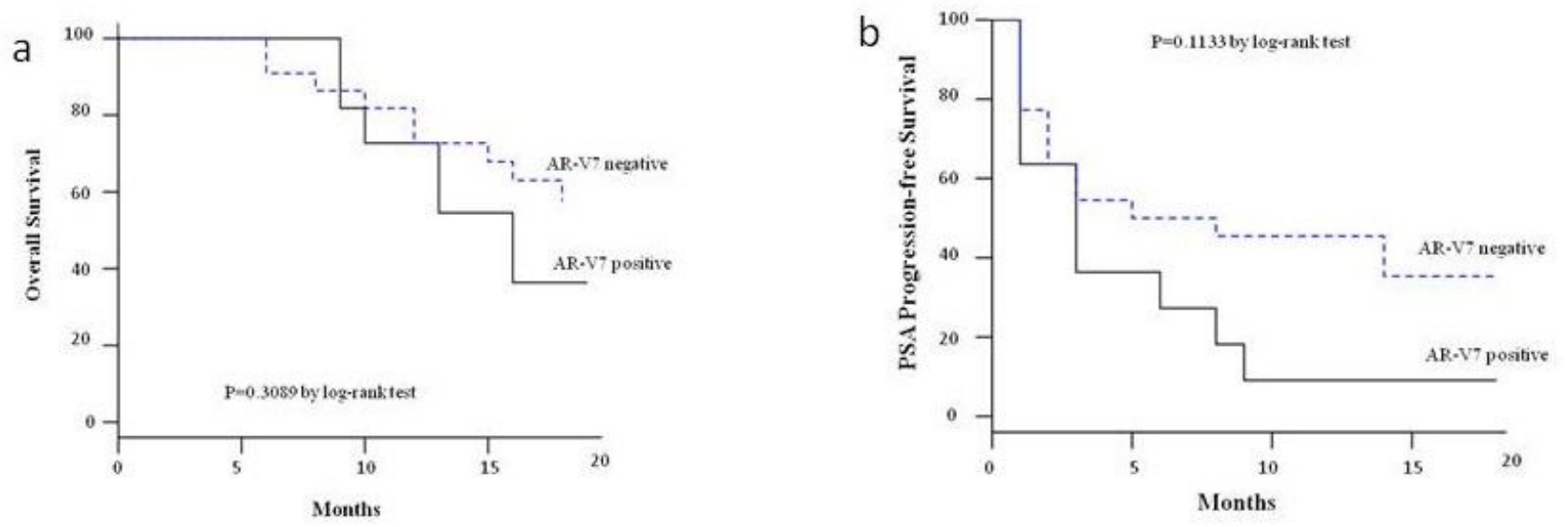

Figure 5 
Overall survival and PSA progression-free survival curves according to AR-V7 analysis Fig. a Overall survival curve and Fig. b PSA progression-free survival curve display non-significant difference between AR-V7 positive and negative groups probably due to the small series patients enrolled ( $p=0.3089,0.1133$ respectively). However, significant difference was observed when PSA progression rate and survival rate at the follow-up endpoint $(p=0.0031$, 0.0012 respectively by chi-square test).

\section{Supplementary Files}

This is a list of supplementary files associated with this preprint. Click to download.

- Additionalfile.pdf 